\title{
In Silico Study of Anti-Insomnia Mechanism for Suanzaoren Prescription
}

\section{OPEN ACCESS}

Edited by:

Shi-Bing Su,

Shanghai University of Traditional

Chinese Medicine, China

Reviewed by:

Yun K. Tam,

Sinoveda Canada Inc., Canada

Feng Zhu,

Zhejiang University, China

Lin Tao,

Hangzhou Normal University,

China

*Correspondence:

Xue Yang

yxkx1@163.com

Zhiwei Cao

zwcao@tongji.edu.cn

Specialty section:

This article was submitted to

Ethnopharmacology,

a section of the journal

Frontiers in Pharmacology

Received: 16 January 2019

Accepted: 22 July 2019

Published: 22 August 2019

Citation:

Gao J, Wang Q, Huang Y, Tang K, Yang $X$ and Cao $Z$ (2019) In Silico Study of Anti-Insomnia Mechanism

for Suanzaoren Prescription.

Front. Pharmacol. 10:925.

doi: 10.3389/fphar.2019.00925

\author{
Jian Gao ${ }^{1}$, Qiming Wang ${ }^{1}$, Yuwei Huang ${ }^{1}$, Kailin Tang ${ }^{1}$, Xue Yang ${ }^{2 *}$ and Zhiwei Cao ${ }^{1 *}$ \\ ${ }^{1}$ Shanghai 10th People's Hospital, School of Life Sciences and Technology, Tongji University, Shanghai, China, \\ ${ }^{2}$ Department of Traditional Chinese Medicine, Yangpu Hospital, School of Medicine, TongJi University, Shanghai, China
}

Insomnia is a common and widespread sleeping disorder caused by various risk factors. Though beneficial, conventional treatments of insomnia have significant limitations. As an alternative treatment, Chinese herbal formula Suanzaoren prescription (SZRP), composed of Suanzaoren [seeds of Ziziphus jujuba var. spinosa (Bunge) Hu ex H.F.Chow] and four additional herbs, has been reported with significant anti-insomnia effects. Yet the antiinsomnia mechanism of the herb formulae remains unknown. In this study, we attempted to extrapolate the holistic anti-insomnia mechanism of SZRP through herbal targeting and network pharmacology. The results indicated that the ingredients of Suanzaoren can target multi-neurotransmitter receptors at synapse interface, which was reported to be associated with sedative and hypnotic effects, while the four additional herbs can hit multiple pathways downstream of membrane neurotransmitters. Furthermore, the four additional herbs showed highly cooperative targeting patterns in the paralleled and cross-talked pathways related to inflammatory regulation and endocrine system, which may contribute to the additional relief of insomnia caused by inflammation, anxiety, or endocrine disorder. The interesting complementary mechanism we found among the herbal groups of SZRP may provide an example to study Chinese herbal formula and offers clues to future design of anti-insomnia strategy.

Keywords: network pharmacology, suanzaoren prescription, insomnia, bioinformatics, systematic analysis

\section{INTRODUCTION}

Insomnia is a common sleep disorder in which people have difficulty falling asleep or staying asleep (Punnoose et al., 2012). It can sap quality of life and even cause damage to health. Shortterm insomnia can cause daytime sleeping and low energy (Lande and Gragnani, 2010), while long-term insomnia may lead to serious problems including driving accident, anxiety, chronic pain, cardiovascular disease, and heart failure (Taylor et al., 2007). According to its etiology, insomnia can be classified as primary or secondary (National Heart, 2016). Primary insomnia is not directly associated with any other health conditions or problems, and its pathogenesis remains unclear. On the contrary, secondary insomnia is related to those obvious triggers, and it is generally caused by the following reasons: 1) emotional disorder including psychological stress, depression, and anxiety; 2) health conditions of chronic pain, such as arthritis, headache, and other inflammations; 3) hormone disorder including menstruation, menopause, and hyperglycemia (Pepin et al., 2014; Santoro et al., 2015); and 4) some medicines and substances. 
Anti-insomnia treatment often includes lifestyle change, cognitive-behavioral therapy, and prescription medicines (Buysse, 2013). Nonpharmacologic treatments are usually recommended as the first-line treatment (Trauer et al., 2015), while pharmacologic treatments including benzodiazepines and benzodiazepine-like medications are applied as additional treatment if nonpharmacologic treatments fail (Asnis et al., 2015). Though pharmacologic treatments can be effective, patients may not tolerate their side effects such as rebound withdrawal effects, disruption in sleep architecture, grogginess, memory impairment, and undesired behaviors during sleep (Tiller et al., 2003; Wilt et al., 2016). In addition to those drugs mentioned above, antidepressants, antipsychotics, and antihistamines may also be applied for sedative effect. Yet they are usually not recommended in the absence of corresponding disease symptoms. Due to the present limited treatment, a substantial number of patients worldwide have started to seek their sleeping aids in herbal medicine as an alternative approach (Chen et al., 2009; Frass et al., 2012). It has been reported that Chinese herbal formulae "Xiao Yao Wan," "liquorice, wheat, and jujube soup," "Tian Wang Bu Xin Dan," and "Suanzaoren prescription (SZRP)" are clinically effective against insomnia (Chen et al., 2011; Liu et al., 2015; Ko et al., 2016); among them, SZRP is the most widely used and well documented for refractory insomnia. It was first recorded in Shennong Bencao Jing, which is the earliest authoritative monograph on pharmacy in China ( $\mathrm{Gu}, 2007)$. In addition to the clinical practice, highquality randomized controlled trials have confirmed its efficacy and safety (Wang et al., 2010; Wang et al., 2013; Yuan et al., 2013). Moreover, a meta-analysis study covering 1,454 patients showed that both SZRP monotherapy and combinational treatment can improve sleep quality significantly with minimal side effects (Zhou et al., 2018).

SZRP contains the main herb seeds of Ziziphus jujuba var. spinosa (Bunge) $\mathrm{Hu}$ ex H.F.Chow (Suanzaoren) and four additional herbs: Anemarrhena asphodeloides Bunge (Zhimu), Wolfiporia extensa (Peck) Ginns (Fuling), Ligusticum sinense Oliv. (Chuanxiong), and Glycyrrhiza uralensis Fisch. (Zhigancao). According to the TCM theory and Chinese Pharmacopeia (China, 2015), Suanzaore is the Emperor (Jun), Zhimu and Fuling are the Ministers (Chen), Chuanxiong is the Adjuvant (Zuo), and Zhigancao is the Courier (Shi) (WHO, 2007). The functions of herb are usually believed to be multiple because of the vast chemical diversity. Previous literatures have reported that Suanzaoren is anti-insomnia (Shergis et al., 2017), Zhimu is laxative and anti-inflammatory (Park et al., 2018; Ji et al., 2019; Li et al., 2019), Fuling is diuretic (Zhao et al., 2012), and Chuanxiong is anti-migraine (Shan et al., 2018). However, current studies about the pharmacological mechanism of SZRP are mainly focused on the main herb Suanzaoren. Photochemical analysis indicated that Suanzaoren contains flavonoids, saponins, and triterpenes. Jujuboside A, sanjoinine A, and flavonoids in Suanzaoren were reported to have sedative and hypnotic effects. Further study showed that jujuboside A affected GABAergic and serotonergic system in rat through glutamate-mediated excitatory signal pathway (Cao et al., 2010). The hydrolysis product of jujuboside A, jujubogenin, was predicted to have high potential of blood-brain barrier penetration ability (Chen et al., 2008). In addition, sanjoinine A was found to be able to prolong sleeping time through increasing chloride influx and GABA synthesis (Zhang et al., 2003). In addition, the flavonoid 6-hydroxyflavone in Suanzaoren showed GABA agonistic action by binding to GABAA receptors (Ren et al., 2010). These aforementioned studies provide a glimpse of the partial mechanism of anti-insomnia effects of Suanzaoren herb. The holistic mechanism of SZRP formulae remains unclear. What is the mechanism of additional herbs in treating insomnia? How can they help to improve the therapeutic effects of the main herb? No systematic studies have been published so far. In this work, first, we conducted a global analysis of all known targets of herbal ingredients for five herbs of SZRP to elucidate the overall anti-insomnia mechanisms of SZRP, and then we analyzed the targeting network patterns herb by herb for better understanding of their potential roles in TCM formulae.

\section{METHODS}

\section{Dataset}

The information of herbal ingredients and targets was collected from online TCM databases Traditional Chinese Medicine Systems Pharmacology (Ru et al., 2014), Herb Ingredients' Targets (HIT) (Ye et al., 2011), and Natural Product Activity \& Species Source Database (NPASS) (Zeng et al., 2018). The following key words were used to search in these databases: Ziziphus jujuba var. spinosa (Bunge) Hu ex H.F.Chow OR Suanzaoren; Anemarrhena asphodeloides Bunge OR Zhimu; Wolfiporia extensa (Peck) Ginns OR Fuling; Ligusticum sinense Oliv. OR Chuanxiong; and Glycyrrhiza uralensis Fisch. OR Zhigancao. Therapeutic targets were collected from Therapeutic Targets Database (TTD) (Li et al., 2018). The validated information of plants was collected from Kew Royal Botanic Garden (https://mpns.science.kew.org/ mpns-portal/) and The Plant List (http://www.theplantlist.org).

\section{Functional Annotation and Enrichment Analysis}

Statistical analysis of KEGG function enrichment of the target profile was performed by Metascape (Tripathi et al., 2015) (http://metascape.org). The pathways significantly enriched were selected ( $p$-value $<0.01)$. Then only terms with both $-\log$ ( $p$-value) $>5$ and more than $5 \%$ targets falling into the category were retained. The retained terms were mapped into bubble graph by "pyplot" of matplotlib. Finally, bubble graphs of each herb were combined and labeled with Adobe Photoshop software (Adobe, San Jose, California).

\section{Network Construction for SZRP}

To better elaborate the holistic mechanism of SZRP, three subnetworks were compiled by following the procedures: 1) All targets of SZRP were submitted to an online tool KEGG Search Pathway (https://www.genome.jp/kegg/tool/map_ pathway1.html). 2) All result maps of KEGG were downloaded and integrated. 3) Maps related to "nervous," "immune," or 
"endocrine" were reserved. 4) For each label of "nervous," "immune," and "endocrine," multiple pathways were integrated and overlapped according to cross-talk targets in these maps. Detailed information such as targets names and integrated pathways are shown in Table S3. 5) Subnetworks were drawn with Adobe Illustrator software, where intermediate genes were hidden for better display.

\section{RESULTS}

\section{Overall Ingredients and Targets of SZRP}

To understand the potential anti-insomnia mechanism of SZRP holistically, known targets for herbal ingredient in the formula were collected and analyzed. Five medicinal species were validated by botanical documentation (Figure 1). Z. jujube, A. asphodeloides, L. sinense, and G. uralensis were validated by Kew Database and TPL. W. extensa was reported as an edible fungus (Esteban, 2009; Wei et al., 2016). Through database searching, 497 unique targets were collected for five herbs in SZRP (Table 1); 24\% of proteins (119) were reported as therapeutic targets in TTD (Li et al., 2018). Three known therapeutic targets of anti-insomnia drugs were covered by SZRP targets including gamma-aminobutyric acid type A receptor (GABAR), 5-hydroxytryptamine receptor (HTR), and histamine receptor (HRH). All information of targets is shown in Table S1. The distribution of targets of five herbs is shown in Figure 2. Eighty known targets were retrieved for Suanzaoren, among which 26 were shared by all five herbs. In terms of target abundance, $A$. asphodeloides retrieved the most targets, while L. sinense had the most abundant unique targets.
TABLE 1 | Number of ingredients and known targets for each herb in SZRP.

\begin{tabular}{llcc}
\hline Species name & TCM name & Ingredients & Targets \\
\hline $\begin{array}{l}\text { Ziziphus jujuba var. spinosa } \\
\text { (Bunge) Hu ex H.F.Chow }\end{array}$ & Suanzaoren & 35 & 80 \\
Anemarrhena asphodeloides Bunge & Zhimu & 82 & 228 \\
Wolfiporia extensa (Peck) Ginns & Fuling & 58 & 184 \\
Ligusticum sinense Oliv. & Chuanxiong & 292 & 296 \\
Glycyrrhiza uralensis Fisch. & Zhigancao & 272 & 201 \\
Total (SZRP) & & $\mathbf{7 1 1}$ & $\mathbf{4 9 7}$
\end{tabular}

\section{Functional Analysis of Separated SZRP}

To investigate the functional relationship among the five individual herbs of SZRP, targets of each herb were mapped to KEGG for functional enrichment analysis. The top 5 functional pathways for each herb in SZRP are listed in Table 2. It can be seen that apart from the main herb, the top enriched pathway profiles were highly similar for the four additional herbs. In Table S2, the overall pathway enrichment of total formulae is shown with their targets combined. Aside from cellular processes, metabolism, and signal transduction terms, organismal systems of nervous, endocrine, immune infections (NEIs) were significantly enriched. As our aim is to interpolate the potential anti-insomnia mechanism, we tentatively focused on organismal systems of NEIs, which are known to closely relate with insomnia pathology (Santoro et al., 2015; Xiang et al., 2019). To display the functional similarity and difference among herbal groups of SZRP, significant pathway terms of KEGG were mapped into a bubble graph in Figure 3.

The big and higher bubbles in Figure 3 represent those highly significantly enriched pathway terms. It can be seen that the main

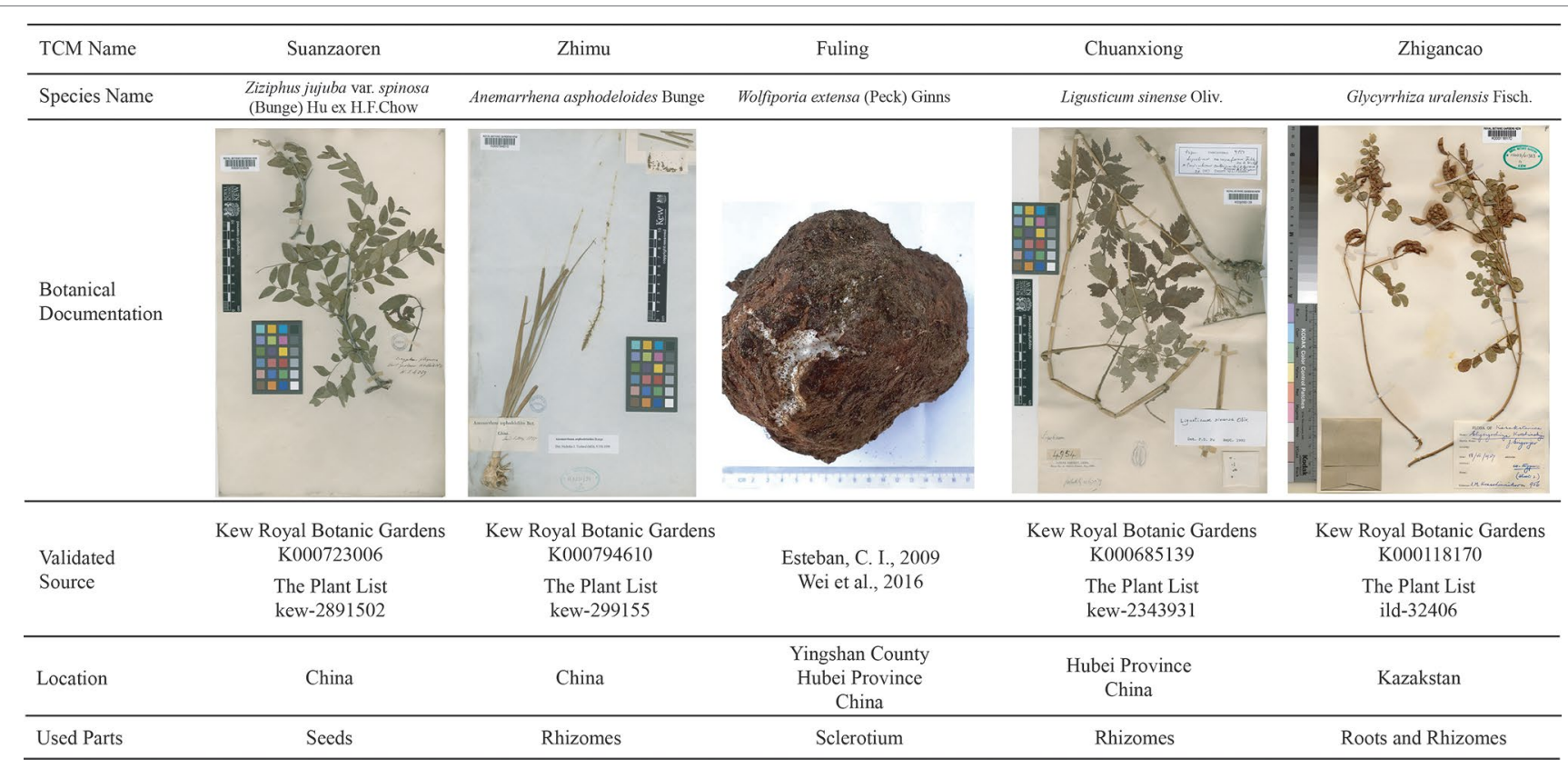

FIGURE 1 | The validated information of five herbs in SZRP, including botanical documentation (voucher specimen deposited in herbarium), location (the collection location of voucher specimen), and used part (used part of medicinal species). 


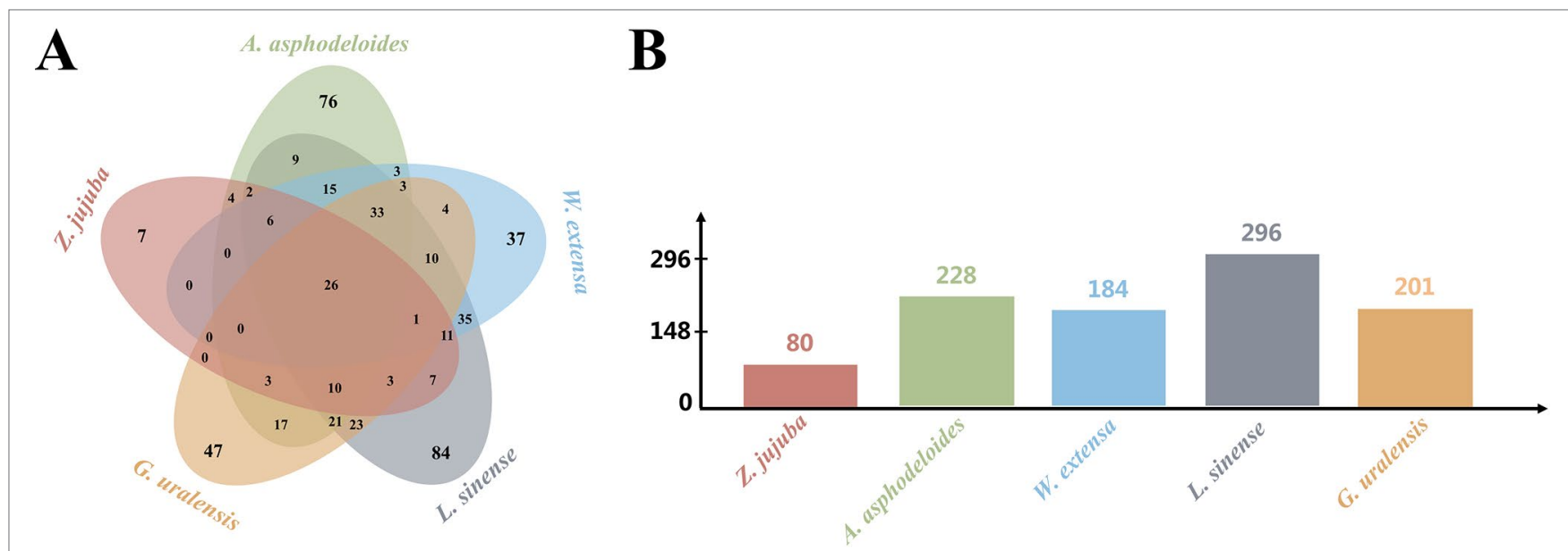

FIGURE 2 | The target distribution of five herbs. (A) Unique and overlapping target of five herbs. (B) The number of targets for different herbs.

TABLE 2 | Top five pathways enriched by targets of each herb in SZRP.

\begin{tabular}{|c|c|c|c|c|c|}
\hline Species name & Herbal group & Pathway name & KEGG ID & Ratio (\%) & -Log(p-value) \\
\hline Ziziphus jujuba var. & Emperor (Jun) & Serotonergic synapse & hsa04726 & 12.5 & 11.09 \\
\hline spinosa & & Dopaminergic synapse & hsa04728 & 10 & 7.7 \\
\hline (Bunge) Hu ex & & Amphetamine addiction & hsa05031 & 7.5 & 6.82 \\
\hline \multirow[t]{2}{*}{ H.F.Chow } & & Adrenergic signaling in cardiomyocytes & hsa04261 & 8.75 & 6.09 \\
\hline & & Cocaine addiction & hsa05030 & 6.25 & 6.08 \\
\hline Anemarrhena & Minister (Chen) & Fluid shear stress and atherosclerosis & hsa05418 & 8.81 & 16.73 \\
\hline \multirow[t]{4}{*}{ asphodeloides Bunge } & & AGE-RAGE signaling pathway & hsa04933 & 6.17 & 11.88 \\
\hline & & Influenza A & hsa05164 & 7.49 & 11.67 \\
\hline & & Ovarian steroidogenesis & hsa04913 & 4.85 & 11.65 \\
\hline & & Adipocytokine signaling pathway & hsa04920 & 5.29 & 11.36 \\
\hline Wolfiporia extensa & Minister (Chen) & Hepatitis B & hsa05161 & 13.66 & 25.55 \\
\hline \multirow[t]{4}{*}{ (Peck) Ginns } & & Influenza A & hsa05164 & 13.11 & 22.1 \\
\hline & & AGE-RAGE signaling pathway & hsa04933 & 9.84 & 18.85 \\
\hline & & Chagas disease (American trypanosomiasis) & hsa05142 & 9.84 & 18.6 \\
\hline & & Hepatitis C & hsa05160 & 10.38 & 17.93 \\
\hline \multirow[t]{5}{*}{ Ligusticum sinense Oliv. } & Adjuvant (Zuo) & AGE-RAGE signaling pathway & hsa04933 & 9.18 & 27.63 \\
\hline & & Hepatitis B & hsa05161 & 9.86 & 25.51 \\
\hline & & Fluid shear stress and atherosclerosis & hsa05418 & 8.84 & 21.77 \\
\hline & & Chagas disease (American trypanosomiasis) & hsa05142 & 7.14 & 18.79 \\
\hline & & Prolactin signaling pathway & hsa04917 & 6.12 & 18.05 \\
\hline Glycyrrhiza uralensis & Courier (Shi) & AGE-RAGE signaling pathway & hsa04933 & 14 & 33.98 \\
\hline \multirow[t]{4}{*}{ Fisch. } & & Fluid shear stress and atherosclerosis & hsa05418 & 15 & 32.19 \\
\hline & & Th17 cell differentiation & hsa04659 & 11.5 & 24.88 \\
\hline & & IL-17 signaling pathway & hsa04657 & 11 & 24.81 \\
\hline & & Hepatitis B & hsa05161 & 11.5 & 21.73 \\
\hline
\end{tabular}

herb of SZRP significantly regulates the function of nervous system, such as dopaminergic and serotonergic synapse pathways, suggesting that the Emperor (Jun) herb may enable hypnotic effects through modulating excitement in nervous system. In contrast, four additional herbs concentrated more significantly on the endocrine system, immunology system, and infections, offering complementary effects against insomnia. Specifically, the Minister (Chen) herb significantly targets virus infection pathways and immune cell pathways, while the Adjuvant (Zuo) and Courier (Shi) herbs affected signaling regulation of oxidative stress (AGE-RAGE) and hormone secretion (Prolactin).

\section{Subnetwork of SZRP on NEI System}

For further investigation, subnetworks were constructed based on enriched pathways for multi-neurotransmitter regulation, inflammatory regulation, and endocrine regulation (Figures 4 and 5). Each target in the pathways was labeled according to the different targeting patterns of the four herbal groups.

Figure 4 shows the targeting patterns of SZRP herbs in a multi-neurotransmitter regulation network. These targets can be roughly divided into three classes: 1) interacted directly with multi-neurotransmitter receptors and downstream pathways; 2) related to the synthesis, secretion, and recycling 


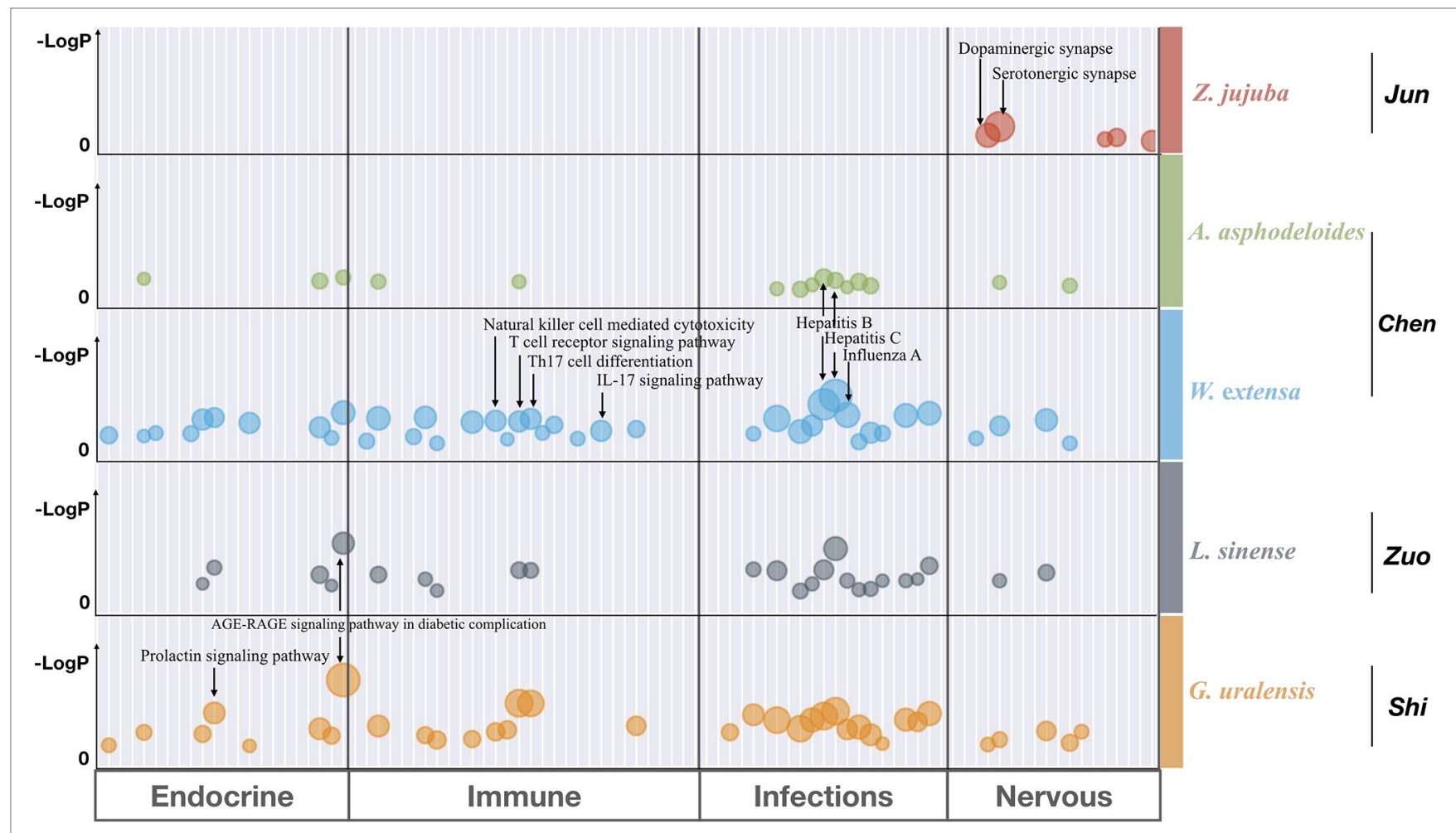

FIGURE 3 | Bubble graph for significantly enriched pathway terms from KEGG by Metascape. Each bubble represents one KEGG pathway term. The size of the bubble correlates with the relative ratio of targets hitting each pathway over the total targets, with larger bubbles indicating the more enriched terms. The LogP shows the statistical significance of $p$-values, with larger and higher numbers indicating the more significant ones. And only those functional terms were retained with both $-\log (p$-value $)>5$ and enrichment ratio $>5 \%$. The detailed statistical data are shown in Table $\mathbf{S 2}$.

of neurotransmitters; and 3) related to the sedative or hypnotic effects, such as opioid receptor (OPR) and adenosine receptor (ADORA). Of interest is that all herbal groups can target transporter proteins of dopamine and serotonin in the presynaptic membrane. When being examined from the perspective of herbal groups, the Emperor (Jun) herb significantly targeted on interfacial neurotransmitter receptors (Figure 4). The four additional herbs not only targeted those receptors but also targeted those in downstream, such as Raf, Akt, PKC, and CaM. Specifically speaking, the Courier (Shi) herb mainly regulated major downstream targets of multi-neurotransmitter regulation pathway. The synapse formation, axonal outgrowth, synaptic plasticity, and neuroprotection medicated by targets nAChR, DR, and HTR may be beneficial to maintain circadian rhythm; in addition, targets of SZRP such as GABRA, ADORA, and HRH have been reported to participate in sleep induction (Huang et al., 2014; Wisden et al., 2017).

Besides, SZRP can regulate immune-related inflammatory pathways and their upstream targets (Figure 5). The Emperor (Jun) herb mainly influenced COX2, while the four additional herbs took part in the regulation of inflammatory factors such as IL-1, IL-2, IL-6, IL-8, and IL-10. And the Courier (Shi) herb covered the largest area of inflammation pathways. Two compounds from the Adjuvant (Zuo) herb L. sinense have been reported to inhibit inflammation through downregulating IL-1 and IL-8 (Diodovich et al., 2003; Medeiros et al., 2007) and upregulating IL-10
(Sarkar et al., 2006). Inflammation and chronic pain are widely reported with insomnia (Cheatle et al., 2016), while inhibition of inflammation and chronic pain was shown to improve sleep quality (Trenkwalder et al., 2017; Wells et al., 2017).

Endocrine disorder associated with premenstrual and menopausal syndromes in women shows outstanding effects on secondary insomnia (Santoro et al., 2015). Figure 5 shows the targeting patterns of SZRP herbs in endocrine regulation pathways. These targets were related to the regulation of blood sugar, blood pressure, and hormone system. All herbal groups mainly exerted a wide range of hormonal regulating effect by targeting the key receptors such as estrogen receptor (ER), progesterone receptor (PR), and adrenergic receptor (ADR). The whole formula played holistic and complementary roles in blood pressure and hormone regulation, while other herbal groups of Minister (Chen), Adjuvant (Zuo), and Courier (Shi) showed unique effects on blood sugar regulation by targeting insulin (INS), insulin receptor (INSR), and glucagon-like peptide 1 receptor (GLP-1R). Menstruationand menopause-related insomnia is often caused by endocrine disorder (Santoro et al., 2015); moreover, hyperglycemia and hypertension are the high-risk factors of insomnia.

To understand the mechanistic relationship within TCM formulae, SZRP was disassembled into individual herbal groups. The Emperor (Jun) herb was found to mainly target receptors in synapse membrane, which might relieve the primary insomnia. The Minister (Chen) herbs can target not only the nervous system 


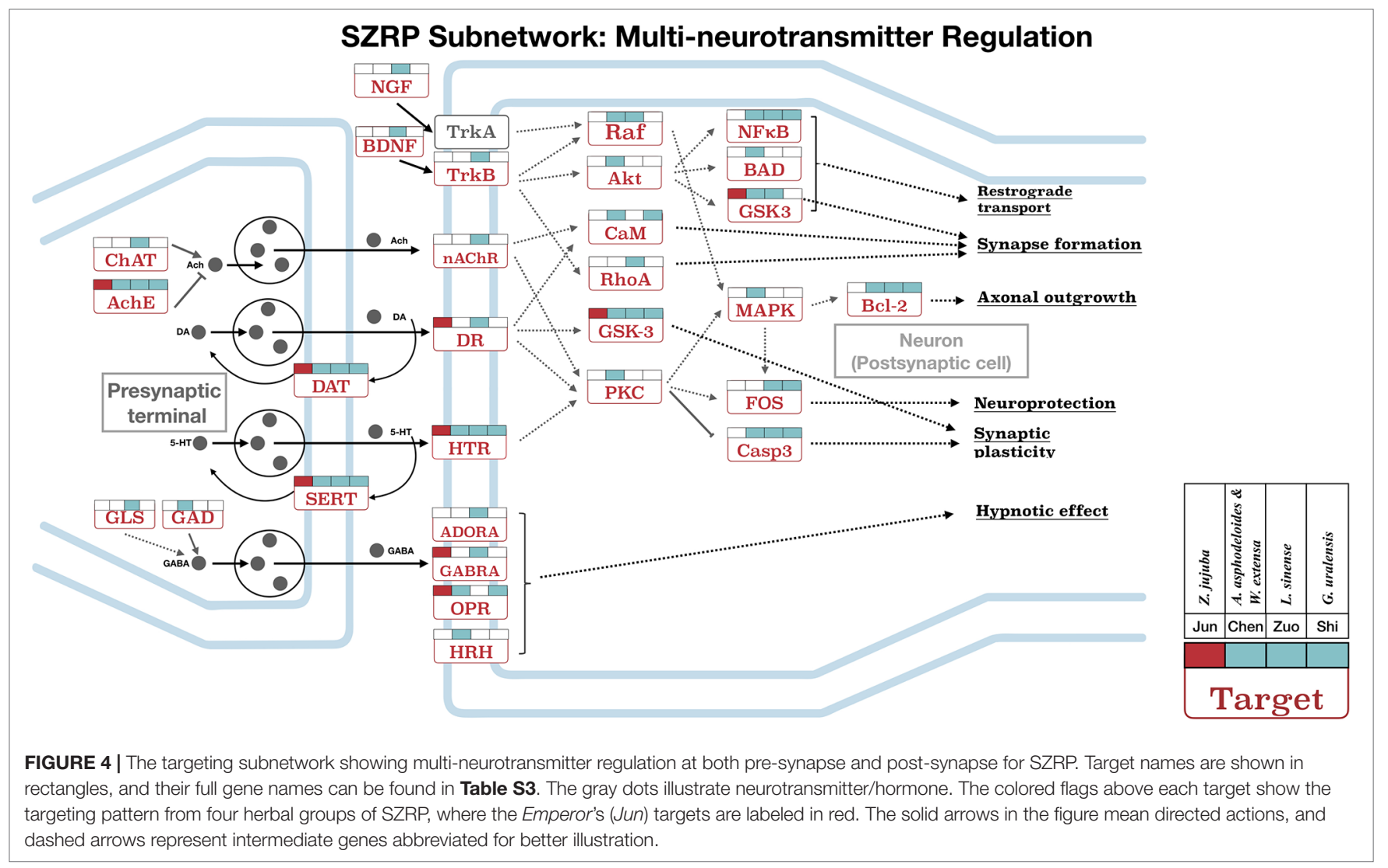

helping the Emperor (Jun) herb but also inflammation and endocrine pathways, providing potential assistance to secondary insomnia. Zhimu has been reported to act on $\mathrm{HRH}$, an effective target for sedative effect (Krystal et al., 2013). Among the herbs, only the Adjuvant (Zuo) herb can upregulate IL-10, the wellknown anti-inflammatory cytokine (Ouyang et al., 2011; Iyer and Cheng, 2012). It is noted that no unique function has been found for the Courier (Shi) herb in the network we constructed. Its function may be relates to the pharmacokinetics. Previous study showed that the Courier (Shi) herb was able to facilitate the adsorption of Suanzaoren (Shen et al., 2012; Bi et al., 2014). These results agree with the TCM theory that Jun, Chen, Zuo, and Shi perform their own functions and cooperate with each other (Fan et al., 2006). In summary, the holistic mechanism of SZRP was first studied in a systematic way by network pharmacology.

\section{DISCUSSION}

In this study, the anti-insomnia mechanism of SZRP was first studied in a distinctive way by networking pharmacology. As a prevalent sleeping disorder, insomnia can be induced by various risk factors. Our research found that multiple pathways were involved in the anti-insomnia effects of SZRP. Five herbs in SZRP seem to play different roles in a complimentary way. The ingredients of the main herb mainly targeted multi-neurotransmitter proteins at the pre-synapse and post-synapse interface exerting sedative and hypnotic effects. This effect was likely enhanced by additional herbs through holistically regulating the nervous system related to insomnia symptoms. On top of that, the additional herbs were suggested to intensely regulate the immune system, particularly inflammation cytokines, which were often reported as an important influential factor for insomnia (Quartana et al., 2015; Irwin et al., 2016; Fernandez-Mendoza et al., 2017). Moreover, the whole formula can target the endocrine system to balance the hormone, blood pressure, and blood sugar, enhancing the sedative and hypnotic effects of the main herb.

By decomposing the formula, the Emperor (Jun) herb, $Z$. jujuba, was found to target synapse membrane, which may fight the main symptom of insomnia. The Minister (Chen) herbs, A. asphodeloides and $W$. extensa, not only can regulate the nervous system helping Jun herb in relieving the main symptom but also can densely target inflammation and endocrine pathways treating the likely pathological causes or secondary symptoms. A. asphodeloides is reported to act on $\mathrm{HRH}$, which is an effective sedative target (Krystal et al., 2013). Interestingly, only the Adjuvant (Zuo) herb can uniquely upregulate IL-10, the wellknown inflammation-inhibiting factor (Ouyang et al., 2011; Iyer and Cheng, 2012) that seems to counteract the inflammationinhibiting effects of other herbal groups. It is noted that no unique function has been found for the Courier (Shi) herb G. uralensis from current MOA networking. We suggest that its possible role in ADME processes cannot be detected in the current analysis (Shen et al., 2012; Bi et al., 2014). The most interesting is to see the targeting patterns in the subnetworks among the herbal groups, such as targeting multi-points of the same pathway, parallel 


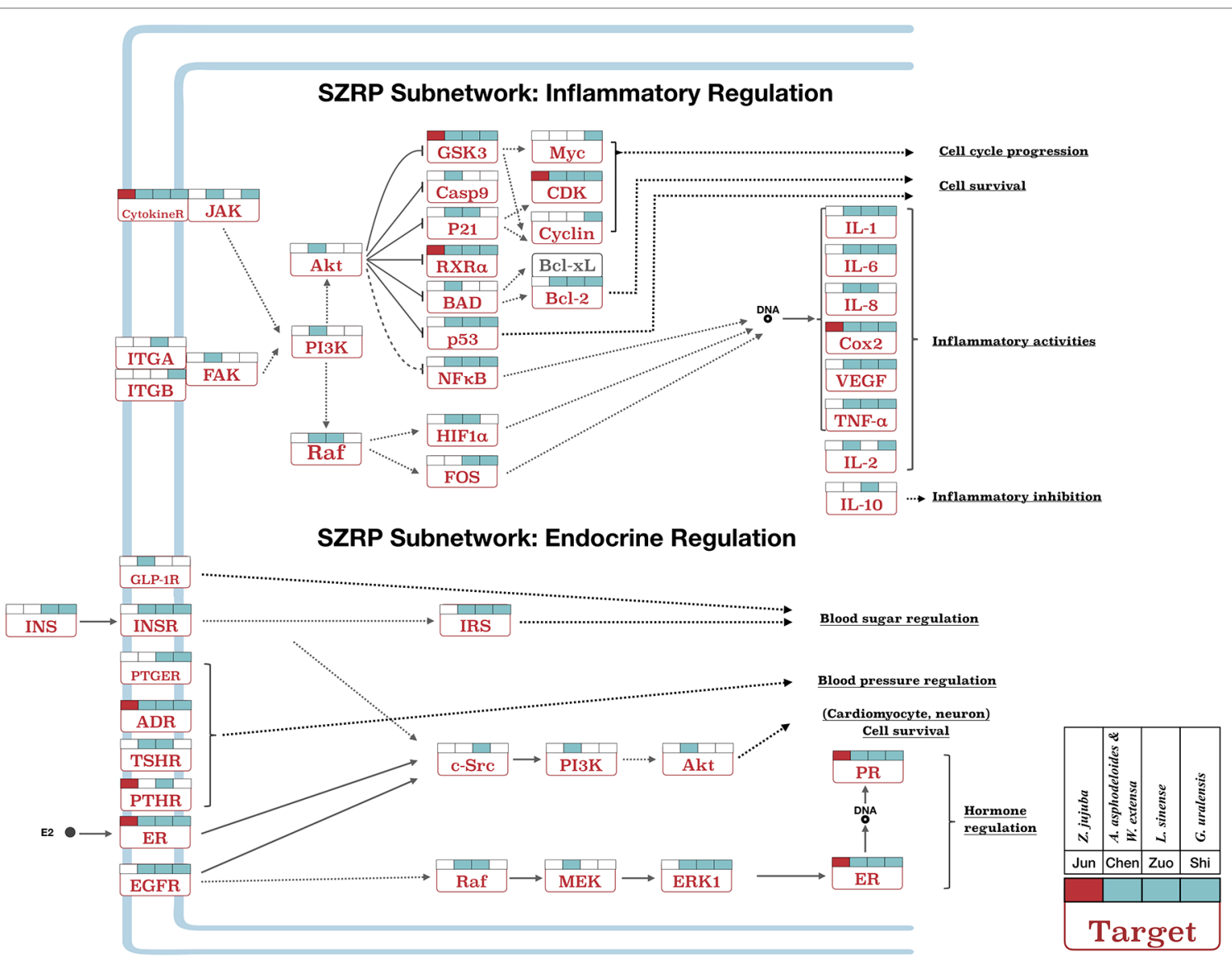

FIGURE 5 | The pathway of inflammatory and endocrine regulation effect in SZRP. Target names are shown in rectangles, and their full gene names can be found in Table S3. All labels are same as Figure 4.

pathways, and cross-talked pathways. These patterns have been reported to have beneficial synergistic effects (Jia et al., 2009) and have been applied to design synergistic drug combinations for cancer (Sun et al., 2015).

Meanwhile, the above results seem to support the TCM theory that the Emperor (Jun) herb often deals with the main symptoms of a disorder, while the Minister (Chen) assists the Emperor (Jun) herb to fight the main symptoms and also to remove those accompanying symptoms and signs. The Adjuvant (Zuo) herbs usually coordinate the effects of the Emperor (Jun) and Minister (Chen) herbs by counteracting their toxic or side effects, and the Courier (Shi) herb helps to deliver or guide the other herbs in the prescription to the target organs (Fan et al., 2006). As such, the future design of anti-insomnia drugs may cover not only the nervous system but also the endocrine and immune systems holistically for better clinical efficacy.

Herbal medicines are usually believed to be multi-functional because of their vast chemical diversity. In this work, we only focused on the widely reported anti-insomnia effects of SZRP. This does not exclude the possibility of additional functions of the formulae. In the pathway enrichment analysis, cancer, cardiovascular disease, signal transformation, and lipid metabolism were also detected for the whole formulae, inferring potential functions to be discovered. In fact, one is often involved with multiple functions and labeled with many different pathways. For instance, NFKB1 was labeled by 67 pathways in KEGG, suggesting its important role as a cross-talk gene. In our case, the ratio of NEI targets chosen for anti-insomnia analysis is about $51.7 \%$ of total unique targets.

It is realized that our study aims to explain the global mechanism of traditional formula by decomposing the herbal formula. The current conclusion may be limited by the data quality and quantity, as well as clinical evidence. To avoid noise induction, we started with a widely acknowledged formula as an example, and their targets with literature support, instead of predicting targets. In the future, more efforts should be devoted to mutual interactions between herbal constituents and the dosage effects. Therefore, we here recommend our method of TCM formula with clear clinical effects and validated targets at the current stage.

\section{AUTHOR CONTRIBUTIONS}

JG wrote the manuscript and contributed to the overall design. QW, YH, and JG analyzed and interpreted the results. YH, KT, 
and QW modified the manuscript. XY and ZC supervised the project. All authors read, critically reviewed, and approved the final manuscript.

\section{FUNDING}

This work was supported in part by the National Key R\&D Program of China (2017YFC1700200 and 2017YFC0908400) and the National Natural Science Foundation of China (31671379).

\section{REFERENCES}

Asnis, G. M., Thomas, M., and Henderson, M. A. (2015). Pharmacotherapy treatment options for insomnia: a primer for clinicians. Int. J. Mol. Sci. 17 (1), 50. doi: 10.3390/ijms17010050

Bi, X., Gong, M., and Di, L. (2014). Review on prescription compatibility of shaoyao gancao decoction and reflection on pharmacokinetic compatibility mechanism of traditional Chinese medicine prescription based on in vivo drug interaction of main efficacious components. Evid. Based Complement. Alternat. Med. 2014, 208129. doi: 10.1155/2014/208129

Buysse, D. J. (2013). Insomnia. JAMA 309 (7), 706-716. doi: 10.1001/jama.2013.193

Cao, J. X., Zhang, Q. Y., Cui, S. Y., Cui, X. Y., Zhang, J., Zhang, Y. H., et al. (2010). Hypnotic effect of jujubosides from Semen Ziziphi Spinosae. J. Ethnopharmacol. 130 (1), 163-166. doi: 10.1016/j.jep.2010.03.023

Cheatle, M. D., Foster, S., Pinkett, A., Lesneski, M., Qu, D., and Dhingra, L. (2016). Assessing and managing sleep disturbance in patients with chronic pain. Anesthesiol. Clin. 34 (2), 379-393. doi: 10.1016/j.anclin.2016.01.007

Chen, C. Y., Chen, Y. F., and Tsai, H. Y. (2008). What is the effective component in suanzaoren decoction for curing insomnia? Discovery by virtual screening and molecular dynamic simulation. J. Biomol. Struct. Dyn. 26 (1), 57-64. doi: 10.1080/07391102.2008.10507223

Chen, F. P., Jong, M. S., Chen, Y. C., Kung, Y. Y., Chen, T. J., Chen, F. J., et al. (2011). Prescriptions of Chinese Herbal medicines for insomnia in Taiwan during 2002. Evid. Based Complement. Alternat. Med. 2011, 236341. doi: 10.1093/ ecam/nep018

Chen, L. C., Chen, I. C., Wang, B. R., and Shao, C. H. (2009). Drug-use pattern of Chinese herbal medicines in insomnia: a 4-year survey in Taiwan. J. Clin. Pharm. Ther. 34 (5), 555-560. doi: 10.1111/j.1365-2710.2009.01038.x

Diodovich, C., Malerba, I., Bowe, G., Acquati, F., Bianchi, M. G., Taramelli, R., et al. (2003). Naphthalene exposure: effects on gene expression and proliferation in human cord blood cells. J. Biochem. Mol. Toxicol. 17 (5), 286-294. doi: 10.1002/ jbt.10091

Esteban, C. I. (2009). Medicinal interest of Poria cocos (= Wolfiporia extensa). Rev. Iberoam. Micol. 26 (2), 103-107. doi: 10.1016/S1130-1406(09)70019-1

Fan, T. P., Yeh, J. C., Leung, K. W., Yue, P. Y., and Wong, R. N. (2006). Angiogenesis: from plants to blood vessels. Trends Pharmacol. Sci. 27 (6), 297-309. doi: 10.1016/j.tips.2006.04.006

Fernandez-Mendoza, J., Baker, J. H., Vgontzas, A. N., Gaines, J., Liao, D., and Bixler, E. O. (2017). Insomnia symptoms with objective short sleep duration are associated with systemic inflammation in adolescents. Brain Behav. Immun. 61, 110-116. doi: 10.1016/j.bbi.2016.12.026

Frass, M., Strassl, R. P., Friehs, H., Mullner, M., Kundi, M., and Kaye, A. D. (2012). Use and acceptance of complementary and alternative medicine among the general population and medical personnel: a systematic review. Ochsner J. 12 (1), 45-56. doi: 10.1043/1524-5012-12.1.45

Gu, G. (2007). Shennong's classic of materia medica, annotated by PJ Yang. Beijing: Academy Press.

Huang, Z. L., Zhang, Z., and Qu, W. M. (2014). Roles of adenosine and its receptors in sleep-wake regulation. Int. Rev. Neurobiol. 119, 349-371. doi: 10.1016/ B978-0-12-801022-8.00014-3

Irwin, M. R., Olmstead, R., and Carroll, J. E. (2016). Sleep disturbance, sleep duration, and inflammation: a systematic review and meta-analysis of cohort

\section{SUPPLEMENTARY MATERIAL}

The Supplementary Material for this article can be found online at: https://www.frontiersin.org/articles/10.3389/fphar.2019.00925/ full\#supplementary-material

TABLE S1 | 497 targets of 5 herbs in SZRP.

TABLE S2 | Top 50 pathways of functional enrichment analysis.

TABLE S3 | Original information of targets in Figure 4-5.

studies and experimental sleep deprivation. Biol. Psychiatry 80 (1), 40-52. doi: 10.1016/j.biopsych.2015.05.014

Iyer, S. S., and Cheng, G. (2012). Role of interleukin 10 transcriptional regulation in inflammation and autoimmune disease. Crit. Rev. Immunol. 32 (1), 23-63. doi: 10.1615/CritRevImmunol.v32.i1.30

Ji, K. Y., Kim, K. M., Kim, Y. H., Im, A. R., Lee, J. Y., Park, B., et al. (2019). The enhancing immune response and anti-inflammatory effects of Anemarrhena asphodeloides extract in RAW 264.7 cells. Phytomedicine 59, 152789. doi: 10.1016/j.phymed.2018.12.012

Jia, J., Zhu, F., Ma, X., Cao, Z., Cao, Z. W., Li, Y., et al. (2009). Mechanisms of drug combinations: interaction and network perspectives. Nat. Rev. Drug Discov. 8 (2), 111-128. doi: 10.1038/nrd2683

Ko, C. H., Koon, C. M., Yu, S. L., Lee, K. Y., Lau, C. B., Chan, E. H., et al. (2016). Hypnotic effects of a novel anti-insomnia formula on Drosophila insomnia model. Chin. J. Integr. Med. 22 (5), 335-343. doi: 10.1007/s11655-014-1625-1

Krystal, A. D., Richelson, E., and Roth, T. (2013). Review of the histamine system and the clinical effects of $\mathrm{H} 1$ antagonists: basis for a new model for understanding the effects of insomnia medications. Sleep Med. Rev. 17 (4), 263-272. doi: 10.1016/j.smrv.2012.08.001

Lande, R. G., and Gragnani, C. (2010). Nonpharmacologic approaches to the management of insomnia. J. Am. Osteopath. Assoc. 110 (12), 695-701

Liu, L., Liu, C., Wang, Y., Wang, P., Li, Y., and Li, B. (2015). Herbal medicine for anxiety, depression and insomnia. Curr. Neuropharmacol. 13 (4), 481-493. doi: 10.2174/1570159X1304150831122734

Li, X., Liu, Y., Guan, W., Xia, Y., Zhou, Y., Yang, B., et al. (2019). Physicochemical properties and laxative effects of polysaccharides from Anemarrhena asphodeloides Bge. in loperamide-induced rats. J. Ethnopharmacol. 240, 111961. doi: 10.1016/j.jep.2019.111961

Li, Y. H., Yu, C. Y., Li, X. X., Zhang, P., Tang, J., Yang, Q., et al. (2018). Therapeutic target database update 2018: enriched resource for facilitating bench-to-clinic research of targeted therapeutics. Nucleic Acids Res. 46 (D1), D1121-D1127. doi: 10.1093/nar/gkx1076

Medeiros, R., Passos, G. F., Vitor, C. E., Koepp, J., Mazzuco, T. L., Pianowski, L. F., et al. (2007). Effect of two active compounds obtained from the essential oil of Cordia verbenacea on the acute inflammatory responses elicited by LPS in the rat paw. Br. J. Pharmacol. 151 (5), 618-627. doi: 10.1038/ sj.bjp. 0707270

National Heart, L.a.B.I. (2016). What is insomnia [Online]. Available: https://www. nhlbi.nih.gov/health-topics/insomnia [Accessed].

Ouyang, W., Rutz, S., Crellin, N. K., Valdez, P. A., and Hymowitz, S. G. (2011). Regulation and functions of the IL-10 family of cytokines in inflammation and disease. Annu. Rev. Immunol. 29, 71-109. doi: 10.1146/ annurev-immunol-031210-101312

Park, B. K., So, K. S., Ko, H. J., Kim, H. J., Kwon, K. S., Kwon, Y. S., et al. (2018). Therapeutic potential of the rhizomes of Anemarrhena asphodeloides and timosaponin A-III in an animal model of lipopolysaccharide-induced lung inflammation. Biomol. Ther. (Seoul) 26 (6), 553-559. doi: 10.4062/biomolther.2017.249

Pepin, J. L., Borel, A. L., Tamisier, R., Baguet, J. P., Levy, P., and Dauvilliers, Y. (2014). Hypertension and sleep: overview of a tight relationship. Sleep Med. Rev. 18 (6), 509-519. doi: 10.1016/j.smrv.2014.03.003

Punnoose, A. R., Golub, R. M., and Burke, A. E. (2012). JAMA patient page. Insomnia. JAMA 307 (24), 2653. doi: 10.1001/jama.2012.6219 
Quartana, P. J., Finan, P. H., Page, G. G., and Smith, M. T. (2015). Effects of insomnia disorder and knee osteoarthritis on resting and pain-evoked inflammatory markers. Brain Behav. Immun. 47, 228-237. doi: 10.1016/j.bbi.2014.12.010

Ren, L., Wang, F., Xu, Z., Chan, W. M., Zhao, C., and Xue, H. (2010). GABAA receptor subtype selectivity underlying anxiolytic effect of 6-hydroxyflavone. Biochem. Pharmacol. 79 (9), 1337-1344. doi: 10.1016/j.bcp.2009.12.024

Ru, J., Li, P., Wang, J., Zhou, W., Li, B., Huang, C., et al. (2014). TCMSP: a database of systems pharmacology for drug discovery from herbal medicines. J. Cheminform. 6, 13. doi: 10.1186/1758-2946-6-13

Santoro, N., Epperson, C. N., and Mathews, S. B. (2015). Menopausal symptoms and their management. Endocrinol. Metab. Clin. North Am. 44 (3), 497-515. doi: 10.1016/j.ecl.2015.05.001

Sarkar, S., Khan, M. F., Kaphalia, B. S., and Ansari, G. A. (2006). Methyl palmitate inhibits lipopolysaccharide-stimulated phagocytic activity of rat peritoneal macrophages. J. Biochem. Mol. Toxicol. 20 (6), 302-308. doi: 10.1002/jbt.20150

Shan, C. S., Xu, Q. Q., Shi, Y. H., Wang, Y., He, Z. X., and Zheng, G. Q. (2018). Chuanxiong formulae for migraine: a systematic review and meta-analysis of high-quality randomized controlled trials. Front. Pharmacol. 9, 589. doi: 10.3389/fphar.2018.00589

Shen, L., Hu, R. W., Lin, X., Cong, W. J., Hong, Y. L., Feng, Y., et al. (2012). Pharmacokinetics of characteristic effective ingredients from individual and combination Shaoyao and Gancao treatment in rats using HPLC fingerprinting. Eur. J. Drug Metab. Pharmacokinet. 37 (2), 133-140. doi: 10.1007/s13318-011-0052-y

Shergis, J. L., Ni, X., Sarris, J., Zhang, A. L., Guo, X., Xue, C. C., et al. (2017). Ziziphus spinosa seeds for insomnia: a review of chemistry and psychopharmacology. Phytomedicine 34, 38-43. doi: 10.1016/j.phymed.2017.07.004

Sun, Y., Sheng, Z., Ma, C., Tang, K., Zhu, R., Wu, Z., et al. (2015). Combining genomic and network characteristics for extended capability in predicting synergistic drugs for cancer. Nat. Commun. 6, 8481. doi: 10.1038/ncomms9481

Taylor, D. J., Mallory, L. J., Lichstein, K. L., Durrence, H. H., Riedel, B. W., and Bush, A. J. (2007). Comorbidity of chronic insomnia with medical problems. Sleep 30 (2), 213-218. doi: 10.1093/sleep/30.2.213

Tiller, J. W. (2003). The management of insomnia: an update. Available: https://www.nps.org.au/australian-prescriber/articles/the-managementof-insomnia-an-update

Trauer, J. M., Qian, M. Y., Doyle, J. S., Rajaratnam, S. M., and Cunnington, D. (2015). Cognitive behavioral therapy for chronic insomnia: a systematic review and meta-analysis. Ann. Intern. Med. 163 (3), 191-204. doi: 10.7326/M14-2841

Trenkwalder, C., Zieglgansberger, W., Ahmedzai, S. H., and Hogl, B. (2017). Pain, opioids, and sleep: implications for restless legs syndrome treatment. Sleep Med. 31, 78-85. doi: 10.1016/j.sleep.2016.09.017

Tripathi, S., Pohl, M. O., Zhou, Y., Rodriguez-Frandsen, A., Wang, G., Stein, D. A., et al. (2015). Meta- and orthogonal integration of influenza "OMICs" data defines a role for UBR4 in virus budding. Cell Host Microbe 18 (6), 723-735. doi: 10.1016/j.chom.2015.11.002

Wang, S., Liu, J., Ma, G., Lan, H., and Zhao, Z. (2013). Clinical study of Zaorenanshen tablet treating insomnia. Hebei J. Tradit. Chin. Med. 35 (8), 1217-1219. doi: 10.3969/j.issn.1002-2619.2013.08.058

Wang, Z., He, M., and Jiang, J. (2010). Sanqi Granule the treatment of insomnia of deficiency heart blood and stagnant heat type randomized double-blind clinical curative effect observation. Liaoning J. Tradit. Chin. Med. 37 (12), 2289-2292.
Wei, W., Shu, S., Zhu, W., Xiong, Y., and Peng, F. (2016). The kinome of edible and medicinal fungus Wolfiporia cocos. Front. Microbiol. 7, 1495. doi: 10.3389/ fmicb.2016.01495

Wells, A. M., Ridener, E., Bourbonais, C. A., Kim, W., Pantazopoulos, H., Carroll, F. I., et al. (2017). Effects of chronic social defeat stress on sleep and circadian rhythms are mitigated by kappa-opioid receptor antagonism. J. Neurosci. 37 (32), 7656-7668. doi: 10.1523/JNEUROSCI.0885-17.2017

WHO (2007). WHO international standard terminologies on traditional medicine in the Western Pacific Region.

Wilt, T. J., MacDonald, R., Brasure, M., Olson, C. M., Carlyle, M., Fuchs, E., et al. (2016). Pharmacologic treatment of insomnia disorder: an evidence report for a clinical practice guideline by the American College of Physicians. Ann. Intern. Med. 165 (2), 103-112. doi: 10.7326/M15-1781

Wisden, W., Yu, X., and Franks, N. P. (2017). "GABA Receptors and the pharmacology of sleep" in Handbook of Experimental Pharmacology. Berlin, Heidelberg: Springer, 1-26.

Xiang, B., Liu, K., Yu, M., Liang, X., Huang, C., Zhang, J., et al. (2019). Systematic genetic analyses of GWAS data reveal an association between the immune system and insomnia. Mol. Genet. Genomic Med. 7 (7), e00742. doi: $10.1002 / \mathrm{mgg} 3.742$

Ye, H., Ye, L., Kang, H., Zhang, D., Tao, L., Tang, K., et al. (2011). HIT: linking herbal active ingredients to targets. Nucleic Acids Res. 39 (Database issue), D1055-D1059. doi: 10.1093/nar/gkq1165

Yuan, C., Chen, Y., and Li, J. (2013). Adjuvant treatment of insomnia of liveryin deficiency pattern by Meian grannule: a randomized double-blind and placebo-controlled trial. Shanghai J. Tradit. Chin. Med. 47 (5), 57-71. doi: 10.16305/j.1007-1334.2013.05.027

Zeng, X., Zhang, P., He, W., Qin, C., Chen, S., Tao, L., et al. (2018). NPASS: natural product activity and species source database for natural product research, discovery and tool development. Nucleic Acids Res. 46 (D1), D1217-D1222. doi: 10.1093/nar/gkx1026

Zhang, M., Ning, G., Shou, C., Lu, Y., Hong, D., and Zheng, X. (2003). Inhibitory effect of jujuboside A on glutamate-mediated excitatory signal pathway in hippocampus. Planta Med. 69 (8), 692-695. doi: 10.1055/s-2003-42786

Zhao, Y. Y., Feng, Y. L., Du, X., Xi, Z. H., Cheng, X. L., and Wei, F. (2012). Diuretic activity of the ethanol and aqueous extracts of the surface layer of Poria cocos in rat. J. Ethnopharmacol. 144 (3), 775-778. doi: 10.1016/j.jep.2012.09.033

Zhou, Q. H., Zhou, X. L., Xu, M. B., Jin, T. Y., Rong, P. Q., Zheng, G. Q., et al. (2018). Suanzaoren formulae for insomnia: updated clinical evidence and possible mechanisms. Front. Pharmacol. 9, 76. doi: 10.3389/fphar.2018.00076

Conflict of Interest Statement: The authors declare that the research was conducted in the absence of any commercial or financial relationships that could be construed as a potential conflict of interest.

Copyright (C) 2019 Gao, Wang, Huang, Tang, Yang and Cao. This is an open-access article distributed under the terms of the Creative Commons Attribution License (CC BY). The use, distribution or reproduction in other forums is permitted, provided the original author(s) and the copyright owner(s) are credited and that the original publication in this journal is cited, in accordance with accepted academic practice. No use, distribution or reproduction is permitted which does not comply with these terms. 